\title{
Cluster size distributions in particle systems with asymmetric dynamics
}

\author{
O. Pulkkinent and J. Merikoskit \\ Department of Physics, University of Jyväskylä, P.O. Box 35, FIN-40351 Jyväskylä, Finland
}

\begin{abstract}
We present exact and asymptotic results for clusters in the one-dimensional totally asymmetric exclusion process (TASEP) with two different dynamics. The expected length of the largest cluster is shown to diverge logarithmically with increasing system size for ordinary TASEP dynamics and as a logarithm divided by a double logarithm for generalized dynamics, where the hopping probability of a particle depends on the size of the cluster it belongs to. The connection with the asymptotic theory of extreme order statistics is discussed in detail. We also consider a related model of interface growth, where the deposited particles are allowed to relax to the local gravitational minimum.
\end{abstract}

\section{INTRODUCTION}

The totally antisymmetric exclusion process (TASEP) is one of the most studied models of nonequilibrium phenomena and has attracted both physicists and mathematicians. Many of its features, including density profiles and currents, have been solved with periodic and open boundary conditions [1] 3]. In particular, the so-called matrix formulation has provided an elegant way of exploring the properties of the steady state and has been successfully applied to get nontrivial results for the so-called shock solutions and for systems with several species of particles [4,5]. Recently, it has been used to solve the exact large deviation functional for the density profile in the case of open boundaries [6].

TASEP has been used to model free traffic flow [7] and traffic jams induced by a crossing [8]. Traffic interpretation naturally raises a question of queue lengths in the system. Although this problem is equivalent to one-dimensional site percolation in the canonical ensemble, if the steady state with usual exclusion dynamics and periodic boundary conditions is considered, being exactly solvable, it may still give some insight to problems, which are too complicated to solve in more complex situations. The statistics of the longest queue, or particle cluster as we shall call them, is interesting because it measures the size of the large deviations in the system. Largest clusters in percolation in general dimension were recently studied by Bazant [9]. An exact solution, with limiting behaviors, is presented in this article for the one-dimensional exclusion process. In exclusion processes, one has also the possibility of changing the microscopic dynamics, which leads to percolation problems with different types of cluster statistics. For example, a model for 'reckless drivers', who are trying to escape from their chasers is considered in the present article. We show that this dynamics results in Poisson statistics for the cluster sizes, which in turn makes the properties of the largest cluster very different from those in the case of the usual TASEP dynamics.

TASEP is also related to two other well-known nonequilibrium systems, namely interface growth and directed polymers in random media [1]. In particular, the cluster properties are translated to the excursion properties of the growing interface and the largest clusters measure the long excursions before turning back.

This article is organized as follows. In Sec. [1], we present the basic definitions and the mapping between TASEP and the so-called zero-range process. The properties of clusters in stationary TASEP are studied using this mapping in Sec. III. In particular, we present analytical results for the largest particle and hole clusters in Secs. III B and III O. In Sec. IV extremal statistics of clusters for TASEP with the modified dynamics is considered. Finally, in Sec. V we discuss a related model of interface growth.

\section{THE MODEL}

The model system we analyze in Secs. III and IV is the totally asymmetric exclusion process (TASEP) with $n$ particles hopping to the right in a one-dimensional lattice of $N$ sites, where $N$ is an even number, with periodic boundary conditions. For convenience (see below), we first restrict

$$
\rho:=n / N=1-1 / k, \text { where } \mathrm{k}=2,3, \ldots .
$$

The dynamics, however, differs from that of the ordinary TASEP. We introduce another parameter $z=0,1, \ldots, n$, which for $z \geq 1$ makes the hopping probability to depend on the length of the queue behind the particle in question as follows: After randomly picking an occupied site, check how many occupied sites there are in between the chosen 
site and the next empty site. If this number is less than or equal to $z$, the rightmost particle in the cluster makes a move to the right - otherwise nothing happens.

Now the case $z=0$ is clearly the usual TASEP. In the present article, we concentrate mainly on the cluster length properties of the two cases $z=0$ and $z=n$. We shall also comment briefly on a model of interface growth, where the deposited particles always relax to the local minimum, which is equivalent to a certain symmetrization of the $z=n$ case.

\section{A. TASEP and the zero-range process}

Many properties of the exclusion process can be accessed by using the mapping [10,11] to the zero-range process introduced in Ref. [12]. The mass variable $M_{i}, i=1,2, \ldots N-n$, of the zero-range process is the number of particles between $i^{\text {th }}$ and $(i+1)^{\text {th }}$ hole in TASEP. Since in TASEP there are $n$ particles and $N-n$ holes on a ring of $N$ sites, there will be $N-n$ mass variables each attaining integer values from zero to $n$ with the constraint that $\sum_{i=1}^{N-n} M_{i}=n$. A jump of a particle in TASEP corresponds to a change $\left(M_{i}, M_{i+1}\right) \rightarrow\left(M_{i}-1, M_{i+1}+1\right)$ for some $i$. This way TASEP is reduced to the problem with $n$ particles hopping to the right on a lattice of $N-n$ sites, now with multiple occupancy allowed.

This mapping is, however, not one-to-one, because there are $N /(N-n)$ TASEP configuration corresponding to one configuration of the mass variables. For example, the number of all mass variable configurations equals the number of solutions of the equation $M_{1}+M_{2}+\ldots+M_{N-n}=n$ in non-negative integers. This number is $\left(\begin{array}{c}N-1 \\ N-n-1\end{array}\right)$. But $\frac{N}{N-n}\left(\begin{array}{c}N-1 \\ N-n-1\end{array}\right)=\left(\begin{array}{l}N \\ n\end{array}\right)$, which equals the number of all corresponding TASEP configurations, i.e. the number of random walk paths from $(0,0)$ to $(N, N-2 n)$. Note that without the restriction of Eq. (2.1) this relation between the numbers of configurations would have applied on average only.

As discussed in Ref. [10], the joint stationary distribution of the zero-range process corresponding to TASEP with general $z$ is the product of the marginal measures of the individual sites. For the cases $z=0$ and $z=n$, however, the stationary state is particularly simple and can be constructed in a straightforward way.

\section{SIMPLE EXCLUSION PROCESS, $z=0$}

\section{A. Stationary cluster size}

It is general knowledge that the stationary distribution of TASEP on a ring of $N$ sites is the uniform distribution [1] the probability of each distinct configuration being $\left(\begin{array}{l}N \\ n\end{array}\right)^{-1}$. This follows immediately from the fact that the transition probability to a given state equals the transition probability from the state in question to other states, which makes the transition matrix doubly stochastic 13. The mapping from TASEP to the zero-range process being one-to-one up to a multiplicative constant, the stationary distribution of the zero-range process corresponding to $z=0$ is also uniform.

Due to the translational invariance of our model system the mass variables $M_{i}$ are identically distributed and the general behavior is described by local expectations. The stationary distribution for size $X_{i}$ of the $i^{\text {th }}$ cluster in TASEP can then be expressed in terms of the stationary mass variable distribution by

$$
P\left(X_{i}=k\right)=P\left(M_{i}=k \mid M_{i} \geq 1\right) .
$$

Next we use translational invariance and the uniformity of measures to conclude that Eq. (3.1) equals the number of solutions to

$$
M_{1}+M_{2}+\ldots+M_{N-n-1}=n-k
$$

where $M_{i}$ 's are non-negative integers and $k \geq 1$, divided by the difference of $\left(\begin{array}{c}N-1 \\ n\end{array}\right)$ and the number of solutions to Eq. (3.2) with $k=0$. Therefore

$$
P\left(X_{i}=k\right)=\left(\begin{array}{c}
N-k-2 \\
n-k
\end{array}\right)\left(\begin{array}{c}
N-2 \\
n-1
\end{array}\right)^{-1} .
$$

Note that one could have derived this result also by simply counting the random walk paths starting with exactly $k$ steps up and ending with a down step and normalizing this number by the number of random walk paths with the 
first step up and the last step down. From Eq. (3.3) we immediately obtain the limiting distribution for the cluster lengths,

$$
P_{\infty}\left(X_{i}=k\right)=(1-\rho) \rho^{k-1}
$$

in agreement with the fact [1] that in an infinite system size the measure for TASEP is the product measure.

The expected cluster size is

$$
\begin{aligned}
\left\langle X_{i}\right\rangle & =\left(\begin{array}{c}
N-2 \\
n-1
\end{array}\right)^{-1} \sum_{k=1}^{N} k \cdot\left(\begin{array}{c}
N-k-2 \\
n-k
\end{array}\right) \\
& =\frac{1-1 / N}{1-\rho} \longrightarrow \frac{1}{1-\rho}, \quad \text { as } N \rightarrow \infty
\end{aligned}
$$

and its variance is

$$
\begin{aligned}
\operatorname{Var} X_{i}= & \frac{(\rho-1 / N)(1-\rho-1 / N)(1-1 / N)}{(1-\rho)^{2}(1-\rho+1 / N)} \\
& \longrightarrow \frac{\rho}{(1-\rho)^{2}}, \quad \text { as } N \rightarrow \infty .
\end{aligned}
$$

The remarkably simple result of Eq. (3.5) shows that the expected cluster size converges to a finite value and it is the number of clusters that has to diverge in the $N \rightarrow \infty$ limit. This can be also verified by the following direct evaluation. Let us first calculate the probability that there are $k$ clusters in the system, i.e. that there are $k$ occupied sites in the mass picture. If the first site is to be occupied, place $k$ sites on a ring, label one of them 1 and fill them with $n$ identical objects, such that every site gets at least one object, in $\left(\begin{array}{l}n-1 \\ k-1\end{array}\right)$ ways. After that, place $N-n-k$ vacant sites in between the $k$ occupied sites in $\left(\begin{array}{c}N-n-1 \\ k-1\end{array}\right)$ ways. On the other hand, if the site 1 is to be vacant, place $k+1$ sites on a ring, label one those 1 and fill the sites $2,3, \ldots, k$, such that every site gets at least one object, in $\left(\begin{array}{l}n-1 \\ k-1\end{array}\right)$ ways. Then add $N-n-k-1$ vacant sites in between in $\left(\begin{array}{c}N-n-1 \\ k\end{array}\right)$ ways. Thus we get the hypergeometric distribution,

$$
\begin{aligned}
P(\# C=k) & =\frac{\left(\begin{array}{l}
n-1 \\
k-1
\end{array}\right)\left[\left(\begin{array}{c}
N-n-1 \\
k-1
\end{array}\right)+\left(\begin{array}{c}
N-n-1 \\
k
\end{array}\right)\right]}{\left(\begin{array}{c}
N-1 \\
n
\end{array}\right)} \\
& =\frac{\left(\begin{array}{c}
n-1 \\
k-1
\end{array}\right)\left(\begin{array}{c}
N-n \\
k
\end{array}\right)}{\left(\begin{array}{c}
N-1 \\
n
\end{array}\right)},
\end{aligned}
$$

which immediately yields

$$
\langle \# C\rangle=n \frac{1-\rho}{1-1 / N} .
$$

From Eqs. (3.5) and (3.8) we find the intuitively expected relation $\langle \# C\rangle \cdot\left\langle X_{i}\right\rangle=n$.

\section{B. Length of the longest cluster}

The knowledge of expectations of maximal objects is of great importance since it measures the size of large deviations in the system. In this section we study the statistics of the longest cluster, $\max _{i} X_{i}$, in TASEP. In particular, we discuss the finite size and limiting distribution functions, related expectations and the effects due to discreteness of the sample space of the mass variables.

The cumulative distribution function (c.d.f.)

$$
F_{\rho, N}(k):=P\left(\max _{i} X_{i} \leq k\right)=P\left(\max _{1 \leq i \leq N-n} M_{i} \leq k\right)
$$

can be calculated as the ratio of the number of solutions to

$$
M_{1}+M_{2}+\ldots+M_{N-n}=n
$$


in non-negative integers with $M_{i} \leq k$ and the number of its solutions with $M_{i} \leq n, \forall i$, respectively. The first of these two numbers equals the coefficient of the $n^{\text {th }}$ order term in

$$
\left(1+x+\ldots+x^{k}\right)^{N-n}=\left(\frac{1-x^{k+1}}{1-x}\right)^{N-n}
$$

and therefore

$$
F_{\rho, N}(k)=\frac{1}{\left(\begin{array}{c}
N-1 \\
n
\end{array}\right)} \sum_{i=0}^{\mu_{n, N}(k)}(-1)^{i}\left(\begin{array}{c}
N-n \\
i
\end{array}\right)\left(\begin{array}{c}
N-(k+1) i-1 \\
N-n-1
\end{array}\right),
$$

where

$$
\mu_{n, N}(k):=\min \{N-n,\lfloor n /(k+1)\rfloor\}
$$

and $\lfloor\cdot\rfloor$ stands for the integer part. Note that for $k \geq n / 2$ the distribution function takes the simple form

$$
F_{\rho, N}(k)=1-(N-n) \frac{\left(\begin{array}{c}
N-k-2 \\
N-n-1
\end{array}\right)}{\left(\begin{array}{c}
N-1 \\
n
\end{array}\right)} .
$$

The expectation calculated in terms of the c.d.f. is

$$
\begin{aligned}
\left\langle\max _{i} X_{i}\right\rangle & =\sum_{k=1}^{n} k \cdot P\left(\left\{M_{i} \leq k \forall i\right\} \cap\left\{\exists j: M_{j}=k\right\}\right) \\
& =\sum_{k=1}^{n}\left[1-P\left(M_{i} \leq k-1 \forall i\right)\right]
\end{aligned}
$$

so that, after simplifications, it can be written as

$$
\left\langle\max _{i} X_{i}\right\rangle=\frac{1}{\left(\begin{array}{c}
N-1 \\
n
\end{array}\right)} \sum_{k=1}^{n} \sum_{i=1}^{\mu_{n, N}(k-1)}(-1)^{i+1}\left(\begin{array}{c}
N-n \\
i
\end{array}\right)\left(\begin{array}{c}
N-k i-1 \\
N-n-1
\end{array}\right),
$$

where $\mu_{n, N}(k)$ was defined in Eq. (3.13).

\section{The limiting extremal distribution}

It seems difficult to extract the limiting distribution and the type of the divergence of the mean from the exact results of Eqs. (3.12) and (3.16) directly. For that purpose, we next consider an alternative approach based on independence.

Intuitively one would expect that as the system gets larger the weaker become the correlations between the clusters. In fact, it is easy to see that the mass variables are asymptotically independent, in that, for $k$ fixed,

$$
P\left(M_{i_{1}}=a_{1}, M_{i_{2}}=a_{2}, \ldots, M_{i_{k}}=a_{k}\right) \longrightarrow \prod_{l=1}^{k} P_{\infty}\left(M_{i_{l}}=a_{l}\right),
$$

where $i_{l} \neq i_{m}, \forall l, m$, and $N \rightarrow \infty$. Therefore, we can approximate the c.d.f. $F_{\rho, N}(k)$ by using the product measure, i.e.

$$
P\left(\max _{i} X_{i} \leq k\right) \approx P\left(M_{1} \leq k\right)^{N-n} .
$$

By writing

$$
\begin{aligned}
P\left(M_{1} \leq k\right) & =1-\left(\begin{array}{c}
N-k-2 \\
n-k-1
\end{array}\right)\left(\begin{array}{c}
N-1 \\
n
\end{array}\right)^{-1} \\
& \longrightarrow 1-\rho^{k+1} \text { as } N \rightarrow \infty
\end{aligned}
$$


we get in the large $N$ limit with $\rho$ fixed

$$
\begin{aligned}
F_{\rho, N}(k) & \sim\left(1-e^{-(k+1) \log 1 / \rho}\right)^{N(1-\rho)} \\
& \approx e^{-e^{-k \log 1 / \rho+\log N \rho(1-\rho)}}
\end{aligned}
$$

This result is, for $k$ sufficiently large, in agreement with the earlier result for the tail because Eq. (3.14) yields in the large system size limit

$$
F_{\rho, N}(k) \sim 1-N(1-\rho) e^{-(k+1) \log 1 / \rho},
$$

which is simply the first order approximation for Eq. (3.20).

The c.d.f. in Eq. (3.20) can now be expressed in terms of a scaled variable

$$
Z_{\rho, N}:=\frac{\max _{i} X_{i}-\frac{\log N \rho(1-\rho)}{\log 1 / \rho}}{(\log 1 / \rho)^{-1}} .
$$

From the asymptotic theory of the extremes for independent and identically distributed (i.i.d.) variables [14] 15] we then obtain the Gumbel, or Fisher-Tippett, distribution

$$
P\left(Z_{\rho, N} \leq y\right) \sim e^{-e^{-y}} .
$$

Strictly speaking, however, the function on the right hand side is continuous, whereas the c.d.f. on the left must be piecewise constant with an intrinsic thickness $\log 1 / \rho$, so that the relation holds only when $(y+\log N \rho(1-\rho)) / \log (1 / \rho)$ is an integer. In fact, Eq. (3.23) would be the correct result for the continuous exponential parent distribution, whereas it has been shown by Anderson [16] that the discrete geometric distribution does not belong to the domain of attraction of the Gumbel distribution at all, and that Eq. (3.23) should indeed be replaced by

$$
\begin{aligned}
& \limsup _{N \rightarrow \infty} P\left(Z_{\rho, N} \leq y\right) \leq e^{-e^{-y}}, \\
& \liminf _{N \rightarrow \infty} P\left(Z_{\rho, N} \leq y\right) \geq e^{-e^{-y+\log 1 / \rho}},
\end{aligned}
$$

i.e. the limiting distribution function has Gumbel envelopes.

The expectation of a random variable with the Gumbel distribution is known to equal Euler's constant $\gamma \approx 0.5772$ [15], but the evaluation of the expectation of a discrete variable with Gumbel envelopes is not easily found in the literature. A direct calculation using the proper point probability function seems tedious and, therefore, we argue on heuristic grounds that since the piecewise constant c.d.f. of the scaled variable $Z_{\rho, N}$ is confined between two envelope functions with expectations $\gamma$ and $\gamma+\log 1 / \rho$, the final result should be written as

$$
\left\langle\max _{i} X_{i}\right\rangle \approx \frac{\gamma+\log N \rho(1-\rho)}{\log 1 / \rho}+\frac{1}{2},
$$

where $N$ is large. Fig. 1 (a) shows that the exact result Eq. (3.16) and the asymptotic formula Eq. (3.26) are in excellent agreement with the simulation data [17].

The effect of discreteness of the mass variables can be seen even more clearly in the behavior of the variance than of the expectation. For continuous exponential variables, the variance of the maximum converges to a constant $\sigma^{2} \pi^{2} / 6$ [15], where the scaling parameter $\sigma$ equals $(\log 1 / \rho)^{-1}$ in our case. The simulation results of Fig. [1(b) display persistent fluctuations around this value, with the distance between the maxima diverging logarithmically in $N$. This kind of behavior has recently been observed also by Bazant [9] in connection with percolation theory and the limiting distribution functions described by Eqs. (3.24) and (3.25) were called Fisher-Tippett limit cycles because of the quasiperiodic fluctuations of the variance.

\section{TASEP WITH ASYMMETRIC DYNAMICS, $z=n$}

We now turn to the case $z=n$. This means that every time we pick a particle at random, the rightmost particle in the cluster, where the chosen particle belongs to, makes a move. In the mass picture this translates to $n$ independent random walkers on a ring of $N-n$ sites [10]. 


\section{A. Stationary cluster size}

The joint stationary distribution for $n$ independent random walkers on a ring of $N-n$ sites is

$$
P\left(M_{1}=m_{1}, \ldots, M_{N-n}=m_{N-n}\right)=\frac{1}{(N-n)^{n}}\left(\begin{array}{c}
n \\
m_{1} \ldots m_{N-n}
\end{array}\right),
$$

where $M_{i}$ is the number of walkers on the site $i$. By Eq. (3.1), the cluster length distribution for the TASEP variables $X_{i}$ is now

$$
P\left(X_{i}=k\right)=\frac{\left(1-\frac{1}{N(1-\rho)}\right)^{\rho N-k} N^{-k}(1-\rho)^{-k}}{1-\left(1-\frac{1}{N(1-\rho)}\right)^{\rho N}}\left(\begin{array}{c}
N \rho \\
k
\end{array}\right),
$$

the limit of which is Poisson with the proper normalization,

$$
P_{\infty}\left(X_{i}=k\right)=\frac{\lambda^{-k}}{k !\left(e^{\lambda}-1\right)}, \quad \text { where } \lambda=\frac{\rho}{1-\rho} .
$$

The parameter $\lambda$ introduced above describes the length scale of correlations in the system. Remember that in the case $z=0$ the corresponding parameter was $(\log 1 / \rho)^{-1} \geq \lambda$, so that the correlations are expected to die out faster in the present case. Now we have the mean and the variance of the length of particle clusters

$$
\begin{gathered}
\left\langle X_{i}\right\rangle=\frac{\lambda}{1-\left(1-\frac{1}{N(1-\rho)}\right)^{\rho N}} \\
\longrightarrow \frac{\lambda}{1-e^{-\lambda}} \quad \text { as } N \rightarrow \infty, \\
\operatorname{Var} X_{i}=\left\langle X_{i}\right\rangle\left(1+\lambda-\left\langle X_{i}\right\rangle\right) \\
\longrightarrow \frac{\lambda}{1-e^{-\lambda}}\left(1+\lambda-\frac{\lambda}{1-e^{-\lambda}}\right) \quad \text { as } N \rightarrow \infty .
\end{gathered}
$$

Since there are $\left(\begin{array}{c}N-n \\ k\end{array}\right)$ ways to choose $k$ sites from a ring of $N-n$ sites and $k ! S(n, k)$, where $S(n, k)$ is the Stirling number of the second kind, ways to put $n$ particles into $k$ sites such that each site gets at least one particle, the distribution for the number of clusters now reads

$$
P(\# C=k)=\left(\begin{array}{c}
N-n \\
k
\end{array}\right) \frac{k ! S(n, k)}{(N-n)^{n}}
$$

which again gives $\langle \# C\rangle \cdot\left\langle X_{i}\right\rangle=n$.

Due to the lack of particle-hole symmetry, the properties of the hole clusters differ from those presented above. However, the distribution for the length $H_{i}$ of the $i^{\text {th }}$ hole cluster can be easily constructed in terms of the distribution for the mass variables,

$$
\begin{aligned}
P\left(H_{i}=k\right)= & \begin{cases}P\left(M_{i+1}>0\right) \\
P\left(M_{i+1}=0, M_{i+2}=0, \ldots, M_{i+k-1}=0, M_{i+k}>0\right) & \text { if } k \geq 2\end{cases} \\
= & \left(1-\frac{k-1}{N(1-\rho)}\right)^{\rho N}-\left(1-\frac{k}{N(1-\rho)}\right)^{\rho N} \\
& \longrightarrow\left(e^{\lambda}-1\right) e^{-\lambda k} \quad \text { as } N \rightarrow \infty .
\end{aligned}
$$

The corresponding c.d.f is

$$
P\left(H_{i} \leq k\right)=1-\left(1-\frac{k}{N(1-\rho)}\right)^{\rho N} \longrightarrow 1-e^{-\lambda k} \quad \text { as } N \rightarrow \infty,
$$

and the expected length of hole clusters

$$
\left\langle H_{i}\right\rangle=\sum_{k=1}^{N-n}\left(1-\frac{k-1}{N(1-\rho)}\right)^{\rho N} \longrightarrow \frac{1}{1-e^{-\lambda}} \quad \text { as } N \rightarrow \infty .
$$




\section{B. Length of the longest cluster}

The exact longest particle cluster c.d.f. for the case $z=n$ reads

$$
P\left(\max _{i} X_{i} \leq k\right)=\frac{1}{(N-n)^{n}} \sum_{\substack{m_{i} \leq k, \forall i, \sum_{i}^{m_{i}=n}}}\left(\begin{array}{c}
n \\
m_{1} \ldots m_{N-n}
\end{array}\right),
$$

which seems to be, from the viewpoint of applications, even less instructive than Eq. (3.12) was for the ordinary TASEP. However, an approximate form for the tail of the distribution can be obtained from Eq. (4.10) assuming that, for $k$ sufficiently large, at most one of the masses exceeds $k$,

$$
\begin{aligned}
P\left(\exists j: X_{j}>k\right)= & \frac{1}{(N-n)^{n}} \sum_{\substack{\exists j: m_{j}>k, \sum_{i}^{m_{i}=n}}}\left(\begin{array}{c}
n \\
m_{1} \ldots m_{N-n}
\end{array}\right) \\
\approx & (N-n) \sum_{m_{N-n}=k+1}^{n}\left(\begin{array}{c}
n \\
m_{N-n}
\end{array}\right) \frac{(N-n-1)^{n-m_{N-n}}}{(N-n)^{n}} \\
& \times \sum_{m_{i} \leq k, \forall i,}\left(\begin{array}{c}
n-m_{N-n} \\
m_{1} \ldots m_{N-n-1}
\end{array}\right) \frac{1}{(N-n-1)^{n-m_{N-n}}} .
\end{aligned}
$$

Here the sum in the last term is the probability that on a ring of $N-n-1$ sites with a total of $n-m_{N-n}$ independent random walkers there are at most $k$ walkers on a single site. But for $k \geq(n-1) / 2$, and approximately perhaps even for smaller $k$ values, this probability equals unity and therefore we have

$$
\begin{aligned}
P\left(\max _{i} X_{i} \leq k\right) & \approx 1-\sum_{m_{N-n}=k+1}^{n}\left(\begin{array}{c}
n \\
m_{N-n}
\end{array}\right) \frac{(N-n-1)^{n-m_{N-n}}}{(N-n)^{n-1}} \\
& \sim 1-N(1-\rho) \lambda^{k} e^{-\lambda} \sum_{j \geq 0} \frac{\lambda^{j}}{j !(k+j+1) !} \\
& \approx 1-n \frac{\lambda^{k} e^{-\lambda}}{(k+1) !}
\end{aligned}
$$

when $k$ is sufficiently large and also $N$ large at the last two stages.

Again, one can show that the cluster sizes are asymptotically independent, in that Eq. (3.17) holds with

$$
P\left(M_{i}=k\right)=\frac{(N-n-1)^{n-k}}{(N-n)^{n}}\left(\begin{array}{l}
n \\
k
\end{array}\right) \longrightarrow P_{\infty}\left(M_{i}=k\right)=\frac{\lambda^{k} e^{-\lambda}}{k !} \text { as } N \rightarrow \infty .
$$

Therefore, Eq. (4.12) suggests that the limiting distribution function is, as in the $z=0$ case, approximately given by the product

$$
\begin{aligned}
F_{\lambda, n}(k) & \sim\left(1-e^{-\lambda} \sum_{j \geq k+1} \frac{\lambda^{j}}{j !}\right)^{n / \lambda} \\
& \approx e^{-\frac{e^{-\lambda} n}{\lambda} \sum_{j \geq k+1} \frac{\lambda^{j}}{j !}} \\
& \approx e^{-\frac{e^{-\lambda_{\lambda} k} n}{(k+1) !}},
\end{aligned}
$$

where $k>\lambda$ and $N$ is large. From the mathematical point of view, however, one should be careful with this approximation, because the distribution function for the maximum of i.i.d. Poisson variables does not actually converge to the Gumbel distribution - not even in sense of continuous Gumbel envelopes as it was in the $z=0$ case [16, 18 . Namely, Anderson has shown [16] that the probability of the maximum concentrates on two consecutive integers, i.e. there exists a sequence of integers $I_{n}(\lambda)$ such that 


$$
\lim _{n / \lambda \rightarrow \infty} P\left(\max _{1 \leq i \leq n / \lambda} M_{i}=I_{n}(\lambda) \text { or } I_{n}(\lambda)+1\right)=1 .
$$

The sequence $\left(I_{n}\right)$ then obviously determines the type of divergence of the mean. It was shown by Kimber [18] that in the leading order this sequence diverges as

$$
I_{n}(\lambda) \sim \frac{\log n}{\log \log n} .
$$

However, we would like to remark that, despite of its shortcomings, the approximation of Eq. (4.15) yields the same functional form for the expected length of the longest cluster: In the leading order

$$
\left\langle\max _{i} X_{i}\right\rangle \approx \sum_{k=0}^{n-1} \chi_{\left\{k \leq m_{x}\right\}},
$$

where $\chi_{\{\cdot\}}$ is the indicator function and $m_{x}$ is some crossover mass, where the distribution function increases most rapidly. Eq. (4.15) then gives the very interesting result

$$
\frac{\left(m_{x}+1\right) !}{\lambda^{m_{x}}} \sim e^{-\lambda} n
$$

i.e. the expected length of the longest particle cluster diverges as the inverse relation of Eq. (4.19) in $n$. In the special case $\lambda=1$, i.e. $\rho=1 / 2$, the type of divergence reduces to inverse factorial. Furthermore, by Stirling's formula one obtains the same functional form as in Kimber's result in Eq. (4.17). Our analytical result is again supported by simulations as seen in Fig. 2(a).

The plot of the variance in Fig. 2(b) shows again fluctuations, and the divergence of the distance between maxima is now dictated by the inverse relation of Eq. (4.19). In general, it can be seen that the fluctuations are more pronounced than those in the ordinary TASEP. We also observe that the variance has a global maximum at a finite system size. Below this maximum, the correlation length $\lambda$ is comparable to the system size, which results in large fluctuations of the maximum. For $N \gg \lambda$ the system can be considered to consist of a large number of independent copies and the i.i.d. behavior is recovered. In the end, one should notice that, unfortunately, the continuum approximation of the form Eq. (4.15) with the factorial replaced by the gamma function cannot be used to evaluate the variance: In the continuous case, the maximum concentrates on one real number, in that the properly scaled variable converges to a distribution degenerate at zero [14, 16].

In Fig. 2 we show the expectation and the variance also for the hole clusters. According to Eq. (4.7), the limiting distribution for the lengths of the hole clusters is again geometric, as in the case of $z=0$ dynamics, but the number of clusters is random and the length of the longest cluster depends on that number. The solution to this problem can be found by defining new mass variables $M_{i}^{*}$ as the number of holes between $i^{\text {th }}$ and $(i+1)^{\text {th }}$ particle. Eq. (4.8) then tells that

$$
P\left(M_{i}^{*} \leq k\right)=1-C_{\rho, N}\left(1-\frac{k}{N(1-\rho)}\right)^{\rho N} \longrightarrow 1-C_{\rho} e^{-\lambda k} \quad \text { as } N \rightarrow \infty,
$$

where $\left(C_{\rho, N}\right)$ is a sequence of constants with $\lim C_{\rho, N}=C_{\rho}>0$. Since there are now $n$ new mass variables and the parent distribution is geometric-like, the limiting extremal distribution has again Gumbel envelopes and the divergence of the mean is logarithmic, as seen in Fig. 2(a).

\section{DISCUSSION}

We shall now discuss our results from a different point of view. There is a well-known mapping from TASEP to a certain lattice model, namely the one-dimensional single-step model, of interface growth [1]. In this mapping each particle is considered a unit step down and each vacancy a unit step up. The function $h(x, t)$, where $x$ is the spatial and $t$ the time coordinate, obtained this way is defined to be constant between the integers and, say, right-continuous. Clearly, the condition $\rho \neq 1 / 2$ implies existence of a global tilt. The lengths of the clusters in the TASEP picture translate to the lengths of the decreasing parts of the interface. Note that the $z=0$ case has particle-hole symmetry and the results obtained for the decreasing parts (corresponding to particle clusters) are valid for the increasing parts (hole clusters) as well. For $z \geq 1$ the properties of the increasing parts must be calculated from the hole distribution.

The $z \geq 1$ dynamics can be seen to be equivalent to deposition rule such that the deposited particles relax towards the local minima from the decreasing parts of the height function $h(x, t)$. If the particle lands on an increasing part, 
nothing happens. Since this kind of asymmetry is not very common in real applications of the interface interpretation, we also studied numerically a spatial symmetrization of the $z=n$ case with $\rho=1 / 2$. This symmetrization belongs to the Edwards-Wilkinson universality class [19]. In it, deposited particles always flow downhill to a local minimum. In case the particle lands on a hill top, the minimum is determined using a fair coin. The results are plotted in Fig. 3. The mean length of the longest cluster diverges now faster than in the asymmetric $z=n$ case studied in Sec. IV, the earlier analytical result for which is shown for comparison in Fig. 3(a). This is consistent with the fact that on average the hole clusters are considerably shorter than in the case of asymmetric dynamics, which is compensated by longer particle clusters. The other features can be seen to be quite similar to those of the asymmetric case. For example, the variance of the longest cluster has a maximum at finite $N$ and quasiperiodic oscillations as before.

In conclusion, we presented analytical and numerical results for the properties of the clusters in asymmetric exclusion processes with two different dynamics. In particular, we studied the properties of the longest cluster in the system and showed that the large system behavior agrees with the asymptotic theory of the extremes for independent and identically distributed variables. The expected length of the longest cluster was found to diverge logarithmically with increasing system size for the ordinary TASEP and as logarithm divided by double logarithm in the case of modified dynamics, which corresponds to independent random walkers in the mass picture. In the latter case, the length of the longest cluster is distributed among two consecutive integers in the large system limit.

\section{ACKNOWLEDGEMENTS}

We thank Dr. Vesa Ruuska and Prof. Stefan Geiss for discussions. This work has been supported by the Academy of Finland under the Center of Excellence Program (Project No. 44875).

* $\quad$ Corresponding author, Email address: otto.pulkkinen@phys.jyu.fi

$\dagger \quad$ Email address: juha.merikoski@phys.jyu.fi

[1] B. Derrida and M. R. Evans, in Nonequilibrium statistical mechanics in one Dimension, Edited by V. Privman (Cambridge University Press, Cambridge, 1997).

[2] T. M. Liggett, Interacting particle systems (Springer, Berlin, 1985).

[3] T. M. Liggett, Stochastic interacting systems: contact, voter and exclusion processes (Springer, Berlin, 1999).

[4] B. Derrida, M. R. Evans, V. Hakim, and V. Pasquier, J. Phys A 26, 1493 (1993).

[5] B. Derrida, M. R. Evans, and D. Mukamel, J. Phys A 26, 4911 (1993).

[6] B. Derrida, J. L. Lebowitz, and E. R. Speer, cond-mat/0105110.

[7] A. Schadschneider and M. Schreckenberg, J. Phys A 26, L679 (1993).

[8] T. Nagatani, J. Phys A 26, 6625 (1993).

[9] M. Z. Bazant, Phys. Rev. E 62, 1660 (2000).

[10] M. R. Evans, Brazilian J. of Phys. 30, 42 (2000), cond-mat/0007293.

[11] S. N. Majumdar, S. Krishnamurthy and M. Barma, J. Stat. Phys. 99, 1 (2000).

[12] F. Spitzer, Adv. Math. 5, 246 (1970).

[13] P. Brémaud, Markov chains: Gibbs fields, Monte Carlo simulation, and queues (Springer, New York, 1999).

[14] J. Galambos, The asymptotic theory of extreme order statistics (Wiley, New York, 1978).

[15] S. Kotz and S. Nadarajah, Extreme value distributions: theory and applications (Imperial College Press, London, 2000).

[16] C.W. Anderson, J. Appl. Prob. 7, 99 (1970).

[17] All our simulation data was obtained by a standard Monte Carlo procedure using the random number generator RANMAR.

[18] A. C. Kimber, Z. Wahrscheinlichkeitstheorie verw. Gebiete 63, 551 (1980).

[19] P. Meakin, Fractals, scaling and growth far from equilibrium (Cambridge University Press, Cambridge, 1998). 

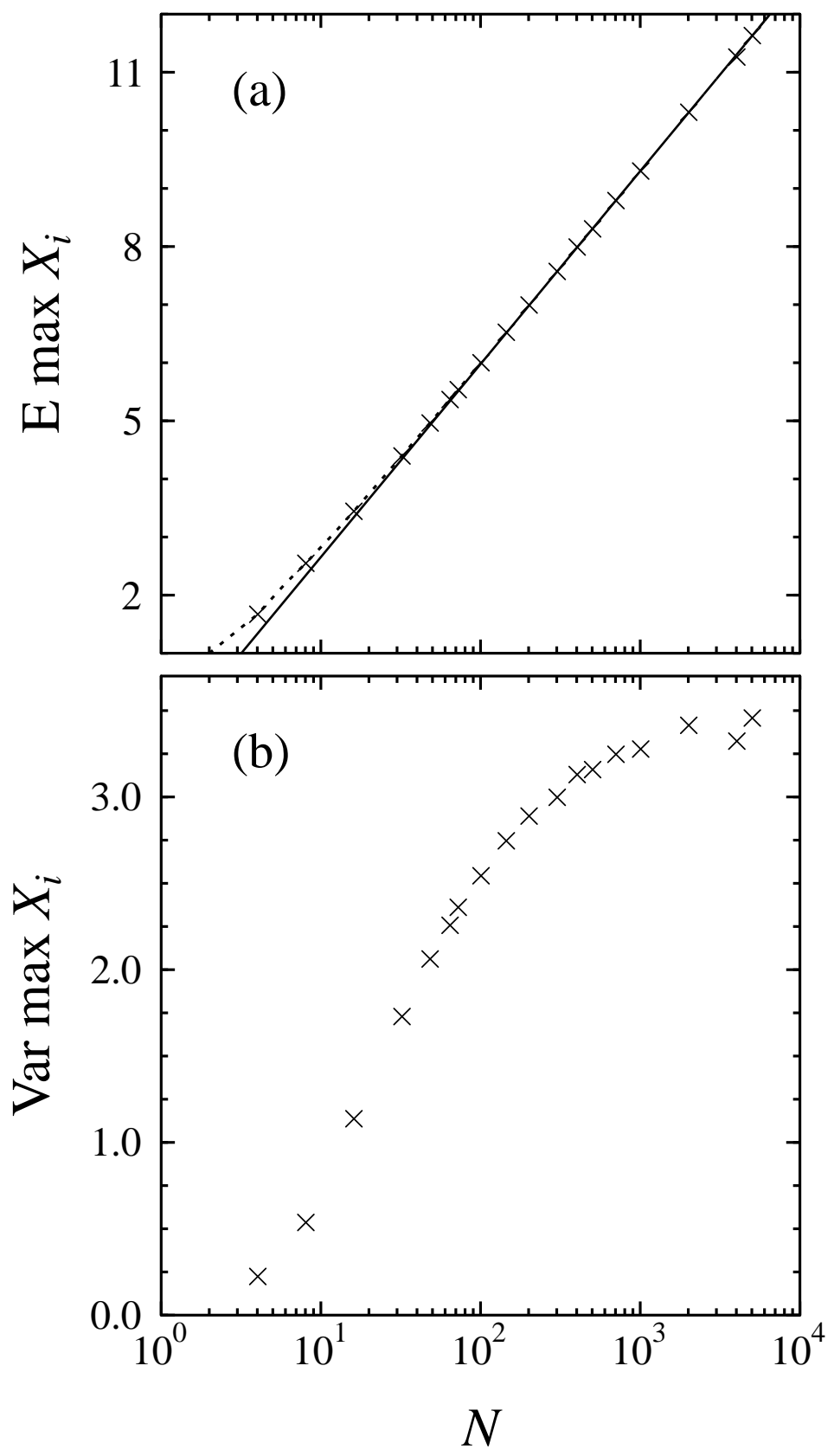

FIG. 1. (a) Expectation value of the size of the longest cluster and (b) its variance as a function of the system size $N$ for $z=0$ with $\rho=1 / 2$. In (a), the dotted curve is the exact result Eq. (3.16) and the full curve the asymptotic formula Eq. (3.26). Crosses denote the simulation data. 

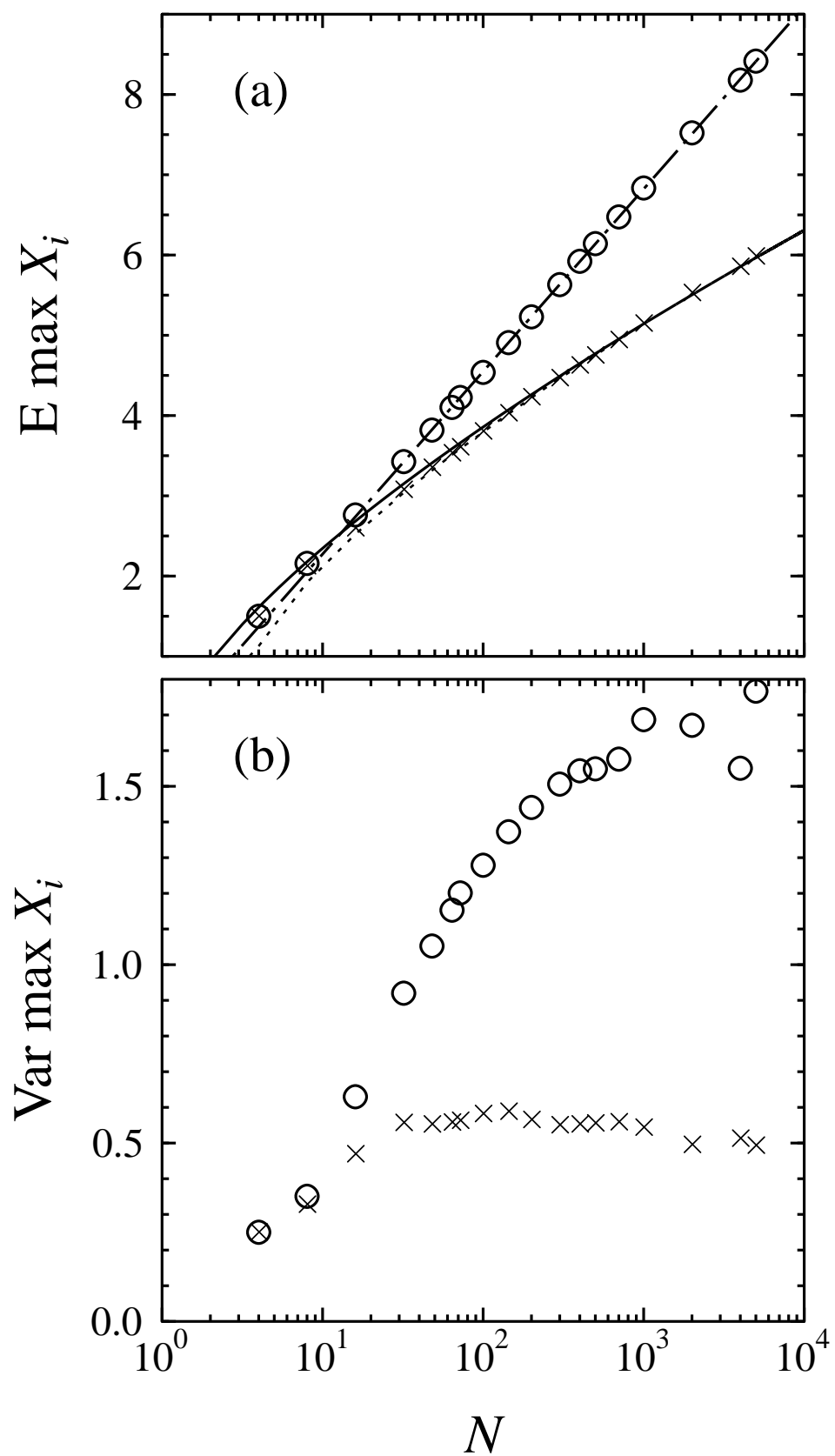

FIG. 2. (a) Expectation values of the size of the longest particle cluster and the size of the longest hole cluster and (b) their variances as a function of the system size $N$ for $z=n$ with $\rho=1 / 2$. Crosses and squares denote the simulation data for particle clusters and hole clusters, respectively. In (a), the dotted curve was obtained by substituting Eq. (4.14) in Eq. (3.15) and the full curve by taking the crossover mass to be the point where the approximate c.d.f. of Eq. (4.15) obtains the value 1/2. The dash-dotted line is the function $0.987 \log N$, where the prefactor was chosen to produce the 'best fit'. 

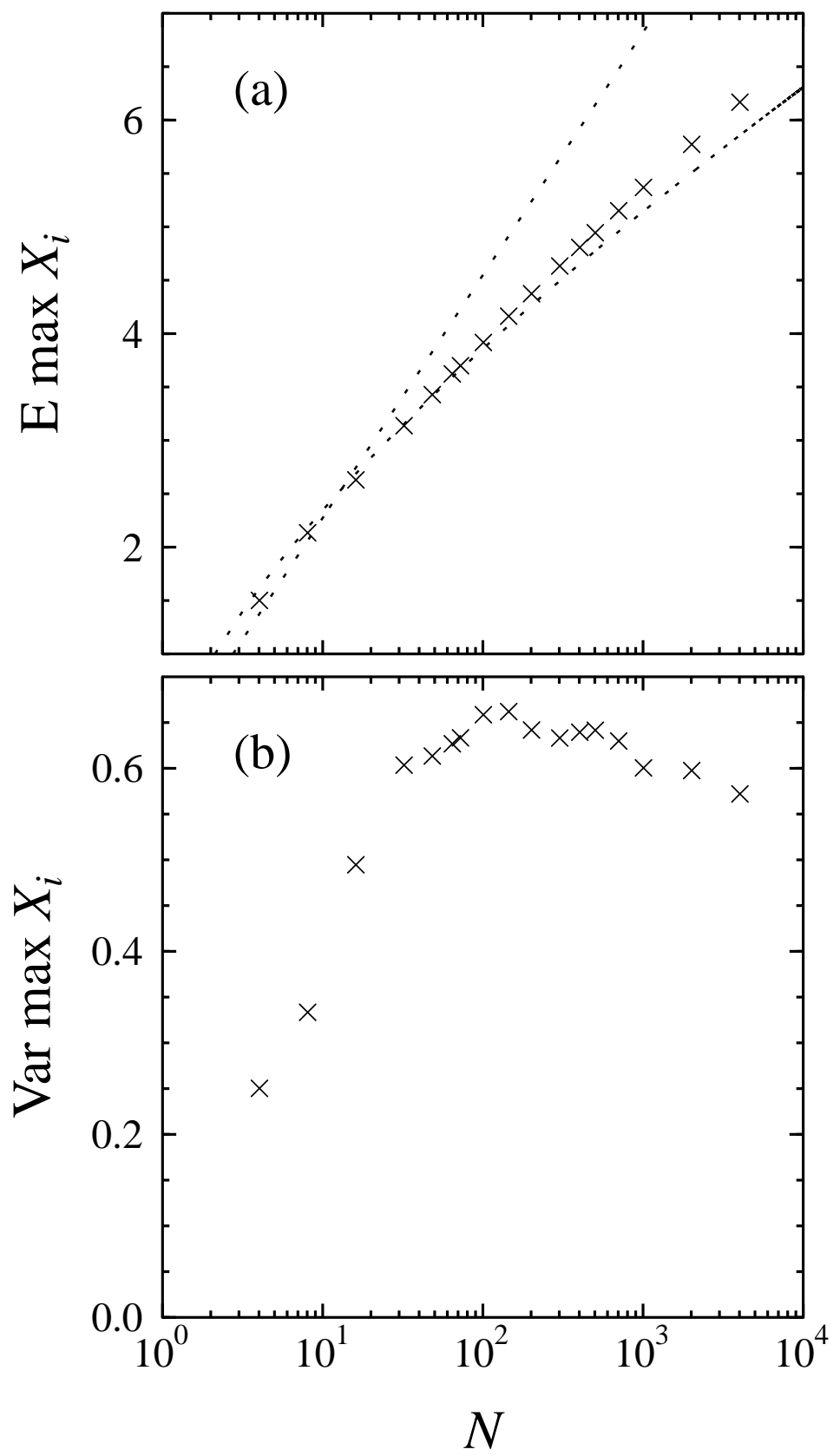

FIG. 3. (a) Expectation values of the size of the longest cluster and (b) its variance as a function of the system size $N$ for the symmetrized model with $z=n$ and $\rho=1 / 2$. Crosses denote the simulation data for clusters in the symmetrized model. In (a), the asymptotic curves for the corresponding asymmetric case from Fig. 2 are shown for comparison by the dashed lines. 